\title{
Food portion sizes and their relationship with dietary energy density in Irish adults
}

Reduction in portion size, particularly for energy-dense foods, to reduce energy intake and help tackle the obesity epidemic is increasingly addressed in healthy eating guidelines. The current work aimed to describe relationships between the portion sizes of a range of foods commonly consumed by Irish adults $(18-64 y ; n$ 1274) and dietary energy density (DED) on the days the foods were consumed.

The Irish National Adult Nutrition Survey (NANS) [2008-2010] used a 4-day semi-weighed food record to collect dietary intake data from a representative sample of Irish adults. Nutrient intakes were estimated using WISP (Tinuviel Software, Anglesey, UK) which contains data from McCance and Widdowson's The Composition of Foods, $6^{\text {th }}$ Edition $^{(1)}$. For the current work, portion size $(\mathrm{g})$ was defined as the weight of food consumed per eating occasion, and was estimated for each day the food was consumed. Where a food was consumed on more than one occasion on one day, the largest portion size was assigned. Portion size data were split by tertile, stratified by gender and age as appropriate. Mean values for DED ( $\mathrm{kJ} / \mathrm{g})$ were examined across tertiles for the days on which the foods were consumed.

\begin{tabular}{|c|c|c|c|c|c|}
\hline & & $\mathrm{ED}(\mathrm{k}]$ & & & Median portion weights per tertile $(\mathrm{g})$ \\
\hline & $\mathrm{T} 1$ & $\mathrm{~T} 2$ & $\mathrm{~T} 3$ & & \\
\hline White bread \& rolls & $7 \cdot 59^{\mathrm{a}}$ & $8 \cdot 01^{\mathrm{b}}$ & $8 \cdot 36^{\mathrm{c}}$ & $\uparrow$ & $50,76,125$ \\
\hline Brown bread \& rolls & $7 \cdot 02$ & $7 \cdot 25$ & $7 \cdot 26$ & & $48,76,114$ \\
\hline Boiled potatoes & $6 \cdot 66^{\mathrm{a}}$ & $6 \cdot 50^{\mathrm{a}}$ & $6 \cdot 11^{\mathrm{b}}$ & $\downarrow$ & $112,181,280$ \\
\hline Ready-to-eat breakfast cereals & $7 \cdot 58^{\mathrm{a}}$ & $7 \cdot 51^{\mathrm{a}}$ & $8 \cdot 10^{\mathrm{b}}$ & $\uparrow$ & $30,45,72$ \\
\hline Fruit, excluding dried fruit & $6 \cdot 92^{\mathrm{a}}$ & $6 \cdot 37^{\mathrm{b}}$ & $5 \cdot 83^{\mathrm{c}}$ & $\downarrow$ & $80,130,188$ \\
\hline Vegetables, excluding pulses & $7 \cdot 71^{\mathrm{a}}$ & $6 \cdot 92^{\mathrm{b}}$ & $6 \cdot 23^{\mathrm{c}}$ & $\downarrow$ & $32,67,116$ \\
\hline Baked beans & $7 \cdot 85^{\mathrm{a}}$ & $7 \cdot 33^{\mathrm{ab}}$ & $6 \cdot 92^{b}$ & $\downarrow$ & $62,120,205$ \\
\hline Frying meats (sausages, bacon) & $7 \cdot 63^{\mathrm{a}}$ & $8 \cdot 26^{\mathrm{b}}$ & $8 \cdot 59^{\mathrm{b}}$ & $\uparrow$ & $30,50,106$ \\
\hline Fillets of fish, uncoated & $6 \cdot 66$ & $6 \cdot 23$ & $6 \cdot 27$ & & $71,126,200$ \\
\hline Cheese & $7 \cdot 41^{\mathrm{a}}$ & $7 \cdot 74^{\mathrm{b}}$ & $8 \cdot 08^{\mathrm{c}}$ & $\uparrow$ & $20,36,60$ \\
\hline Butter \& spreads $>59 \%$ fat & $7 \cdot 30^{\mathrm{a}}$ & $7 \cdot 70^{\mathrm{b}}$ & $8 \cdot 21^{\mathrm{c}}$ & $\uparrow$ & $6,12,24$ \\
\hline Low-fat spreads $<38 \%$ fat & $6 \cdot 72$ & $7 \cdot 10$ & $7 \cdot 21$ & & $8,12,24$ \\
\hline Biscuits & $7 \cdot 09^{\mathrm{a}}$ & $7 \cdot 51^{\mathrm{b}}$ & $8 \cdot 12^{\mathrm{c}}$ & $\uparrow$ & $18,30,52$ \\
\hline Chocolate confectionary & $7 \cdot 51^{\mathrm{a}}$ & $7 \cdot 97^{\mathrm{b}}$ & $8 \cdot 42^{\mathrm{c}}$ & $\uparrow$ & $18,35,56$ \\
\hline Sugary sweets & $7 \cdot 15$ & $7 \cdot 68$ & $8 \cdot 33$ & & $8,28,54$ \\
\hline Sugar-sweetened beverages & $7 \cdot 97^{\mathrm{a}}$ & $8.63^{\mathrm{b}}$ & $8.53^{\mathrm{b}}$ & $\uparrow$ & $200,330,500$ \\
\hline
\end{tabular}

DED was higher on the days larger portions of white bread, ready-to-eat breakfast cereals, frying meats, cheese, butter, biscuits, chocolate and sugar-sweetened beverages were consumed, and lower on the days larger portions of boiled potatoes, fruit, vegetables and baked beans were consumed. Findings are consistent with other studies that show high energy-density diets in adults to be characterised by lower fruit and vegetable intake, and higher intakes of energy-dense nutrient-poor foods ${ }^{(2,3)}$. The findings provide an evidence base from which more specific portion size guidance may be developed for Irish adults, particularly if considered in conjunction with previously published data on the typical portion weights of foods consumed by this group ${ }^{(4)}$.

1. Food Standards Agency (2002) McCance \& Widdowson's The Composition of Foods, $6^{\text {th }}$ Ed. Cambridge: Royal Society of Chemistry.

2. Kant AK \& Graubard BI (2005) Int J Obes 29, 950-956.

3. Ledikwe J, Blanck H, Kettel-Khan L et al. (2006) J Am Diet Assoc 106(8), 1172-1180.

4. Lyons J \& Giltinan M (2013) The Irish Food Portion Sizes Database. www.iuna.net 